\title{
L 1274: A multiwavelength study of a dark cloud in the Cep-Cas void
}

\author{
S. Nikolić ${ }^{1,2,3}$, Cs. Kiss ${ }^{1}$, L. E. B. Johansson ${ }^{2}$, J. G. A. Wouterloot ${ }^{4}$, and L. Viktor Tóth ${ }^{1}$ \\ 1 Department of Astronomy of the Loránd Eötvös University, Pázmány Pétér sétány 1/A, 1117 Budapest, \\ Hungary \\ 2 Onsala Space Observatory, 43992 Onsala, Sweden \\ 3 Astronomical Observatory, Volgina 7, 11160 Belgrade, Serbia, Yugoslavia \\ ${ }^{4}$ Radioastronomisches Institut der Universität Bonn, Auf dem Hügel 71, 53121 Bonn, Germany
}

Received 8 November 1999 / Accepted 4 December 2000

\begin{abstract}
We present a multiwavelength study of L 1274, a dark molecular cloud in the Cep-Cas void. From objective prism spectroscopy we derive a distance of $200 \pm 30$ pc to the cloud. CO maps reveal a slightly elongated molecular cloud with a diameter of about $2 \mathrm{pc}$. Maps in ${ }^{12} \mathrm{CO}(1-0),{ }^{13} \mathrm{CO}(1-0)$ and ${ }^{12} \mathrm{CO}(2-1)$ indicate two distinct features: a "main body" and an adjacent "ridge". The kinetic temperature of the CO gas is 10-12 K, calculated by a non-LTE method. The total gas mass of the main body is estimated from the CO data to be $25-$ $45 M_{\odot}$. We find a large difference between $\mathrm{CO}$-inferred gas mass and virial mass, a property usually attributed to HLCs. However, the derived mean density of $\mathrm{H}_{2}$ is at least an order of magnitude larger than typical for HLCs, i.e. similar to ordinary dark cloud cores. We discuss the validity of our mass estimates and the possibility of an external disturbance, e.g. a passage of a supernova induced shock-front.
\end{abstract}

Key words. ISM: clouds - extinction - dust - molecules, individual objects: L 1274

\section{Introduction}

There are many indications that the interstellar medium in the Cepheus-Cassiopeia region $\left(100 \leq \ell \leq 140^{\circ}, 0 \leq\right.$ $\left.b \leq 25^{\circ}\right)$ is highly disturbed due to one or more supernova explosions in the last $10^{6}$ years. The area between the Cepheus and Cassiopeia molecular cloud complexes is virtually free of $\mathrm{CO}$ emission, with the "hole" filled with excess soft X-ray radiation probably caused by a supernova explosion roughly $10^{4}$ years ago at a distance of $300 \mathrm{pc}$ (Grenier et al. 1989). In addition, a ridge of one of the major radio-continuum loops, Loop III (Berkhuijsen 1971), runs along the eastern boundary of the Cepheus cloud complex. It is generally accepted that the origin of radiocontinuum loops are old supernova remnants with ages estimated to $\sim 10^{6}$ years. Loop III has an estimated distance of $150 \mathrm{pc}$ and the projected centre is $l \approx 125^{\circ}, b \approx 15^{\circ}$ (Berkhuijsen 1973). The dust in the Cep-Cas region reflects a violent environment; in the second Galactic quadrant 135 FIR loops have been defined from the $100 \mu \mathrm{m}$ ISSA images (Tóth et al. 1996; Kiss et al. 2001, in prep.). On the sky molecular clouds appear to follow many of those FIR loops (see, e.g., Kiss et al. 2000), supporting

Send offprint requests to: S. Nikolić,

e-mail: silvana@oso. chalmers.se the hypothesis that these loops trace dust and gas swept out by strong stellar winds and/or supernova explosions.

L 1274 is a small dark cloud with estimated area, opacity and centre coordinates of $0.032 \mathrm{deg}^{2}, 4$ and $\alpha=$ $23^{\mathrm{h}} 55^{\mathrm{m}}, \delta=+70^{\circ} 40^{\prime}$ (1950.0), respectively (Lynds 1962). Cloud No. 454 in Taylor et al. (1987) was identified as L 1274 although their coordinates differ by a few arcminutes from the ones in Lynds catalogue. In the ${ }^{13} \mathrm{CO}$ survey of this region by Yonekura et al. (1997) L 1274 is listed as cloud number 109 and is referred to as the $118.1+08.8$ cloud. They derived a peak hydrogen column density of $1.410^{21} \mathrm{~cm}^{-2}$ and a mass of $31 M_{\odot}$ for a cloud size of $18^{\prime} \times 8^{\prime}$ at an assumed distance of $300 \mathrm{pc}$. The cloud was also detected in the all-sky ${ }^{12} \mathrm{CO}$ survey of Dame et al. (1987) as one of many small clouds in the region $115^{\circ} \leq l \leq 125^{\circ}, 5^{\circ} \leq b \leq 15^{\circ}$. Grenier et al. (1989) show a composite map of the nearby $\mathrm{CO}$ emission in Cassiopeia and Cepheus from the all-sky survey integrated from -8 to $+8 \mathrm{~km} \mathrm{~s}^{-1}$, where L 1274 is part of a fairly large cloud complex at $115^{\circ} \leq l \leq 123^{\circ}, 6^{\circ} \leq b \leq 12^{\circ}$. The projected location of L 1274 is close to a peak of excess soft X-ray emission (Grenier et al. 1989).

We have selected L 1274 as a target for studies of physical and chemical conditions in a molecular cloud possibly 
disturbed by shocks. Here we present and discuss optical, infrared, and CO line data.

\section{Observations and data analysis}

\subsection{Optical ${ }^{1}$}

Objective prism images of the region centred at L 1274 were made with the $60 / 90 / 180 \mathrm{~cm}$ Schmidt telescope of the Konkoly Observatory (Piszkéstető mountain station) and an UV transmitting objective prism with a refracting angle of $5^{\circ}$ and $580 \AA / \mathrm{mm}$ linear dispersion at $\mathrm{H}_{\gamma}$. The field of view of the telescope is $19.5 \mathrm{deg}^{2}$. The spectra were recorded on ORWO ZU emulsions with $2 \mathrm{~mm}$ widening. Two sets of images have been taken: two plates with exposures of $30 \mathrm{~min}$ and two plates with $8 \mathrm{~min}$. The observations were carried out in September and October, 1996.

Classifications of the objective prism spectra were made by eye using the set of criteria of the Bonner Spectral Atlas II (Seitter 1975), by comparing our observed spectra with the spectra set of the MK standards presented in the Atlas, made with a prism that had a linear dispersion at $\mathrm{H}_{\gamma}$ of $645 \AA / \mathrm{mm}$. Errors are estimated to be \pm 2 spectral subclasses, which is equivalent to $\pm 0.4^{\mathrm{mag}}$ in the distance modulus for A-type stars. We have classified a total of 508 stars (B, A, F spectral type) and consider our sample to be complete down to $V=12^{\mathrm{mag}}$ (for A type stars). The stars were identified in the GSC using the SKYMAP software (version 2.7). Visual magnitudes of all the classified stars were taken from the HST Guide Star Catalogue CD-ROM Version 1.1. We assumed all stars to be main-sequence objects and assigned absolute magnitudes, $M_{V}$, using values listed by Lang (1992). The corresponding apparent distance moduli, $\rho=m_{V}-M_{V}$, was calculated.

The distance to a dark cloud can be determined by comparing star counts within the area of the cloud and in a nearby extinction-free field (see, e.g., Trumpler \& Weaver 1953). We define $N(V)$ as the number of stars per square degree brighter than $V$ in apparent magnitude. The shape of the cumulative star number-distance modulus relation, $\log _{10} N(V)-\rho$ (the Wolf diagram, Wolf 1923), provides information of the distance and absorption of the clouds along the line of sight. An average excess extinction (absorption caused by the cloud) can be directly read from the Wolf diagram as the separation between the $\log _{10} N_{\mathrm{c}}(V)$ and $\log _{10} N(V)$ curves along the horizontal lines, where $N_{\mathrm{c}}(V)$ refers to the comparison field. In principle, instead of a comparison star field one can use a galactic extinction - free model curve. The latter method has been used for distance determination in several papers, e.g. Kun (1998), Obayashi et al. (1998), Kun et al. (1994) and is applied here too.

1 The optical data were observed and analysed while S. Nikolić and Cs. Kiss were guest observers at the Konkoly Observatory.

\subsection{FIR}

To investigate the FIR properties of the cloud, we used plates extracted from the final Atlas of Sky images ${ }^{2}$ (Infrared Survey Sky Atlas, ISSA, Wheelock et al. 1994) created from the IRAS data. IPAC (Infrared Processing and Analysing Center) has processed these, on-line available, images to a uniform standard, with a pixel size close to $1^{\prime} .5$. The $3^{\circ} \times 3^{\circ}$ ISSA images were centred at $\ell=118^{\circ}$, $b=8^{\circ}$. Due to the low Galactic latitude of L 1274 the $\propto|\sin b|^{-1}$ background emission law could not be used (Boulanger \& Pérault 1988). To remove the background emission we, therefore, used a second order polynomial.

\subsection{Radio}

Onsala 20 m The Onsala Space Observatory's (OSO) 20$\mathrm{m}$ telescope was used during four observing sessions in 1998 and 2000 . We made ${ }^{12} \mathrm{CO}(1-0)$ and ${ }^{13} \mathrm{CO}(1-0)$ maps and observed some positions in $\mathrm{C}^{18} \mathrm{O}(1-0), \mathrm{CS}(2-1)$ and HCN (1-0). The telescope receiver was a SIS mixer with a typical $T_{\text {rec }}=100 \mathrm{~K}$ (SSB) in the frequency range used. We used a 1600-channel correlator with $20 \mathrm{MHz}$ bandwidth (i.e. a velocity resolution of $0.034 \mathrm{~km} \mathrm{~s}^{-1}$ at $110 \mathrm{GHz}$ ). The HPBW of the telescope at $110 \mathrm{GHz}$ is $35^{\prime \prime}$. The pointing was checked by observing several $\mathrm{SiO}$ maser sources and we estimate the pointing uncertainty to be about $3^{\prime \prime} \mathrm{rms}$ in $\mathrm{Az}$ and $\mathrm{El}$. The $\mathrm{CO}$ observations were made in the frequency switching mode with a throw of $7 \mathrm{MHz}$. Other molecules were observed in the beam switching mode. The chopper-wheel method was used for the calibration, and the intensity scale is given in terms of $T_{\mathrm{A}}^{*}$. For the maps we used a grid spacing of $60^{\prime \prime}$. The rms was $0.5-1 \mathrm{~K}, 0.5 \mathrm{~K}$ and $0.02-0.05 \mathrm{~K}$ for ${ }^{12} \mathrm{CO},{ }^{13} \mathrm{CO}$ and the rest of the observed molecules, respectively.

KOSMA 3 m In August 1998 the cloud was mapped in ${ }^{12} \mathrm{CO}(2-1)$ with the KOSMA $3-\mathrm{m}$ telescope (Kramer et al. 1998) on a $1^{\prime}$ grid. The rms was $0.1-0.2 \mathrm{~K}$. In October we made cross - scans through a central position in ${ }^{12} \mathrm{CO}(3-2),{ }^{13} \mathrm{CO}(2-1)$ and $\mathrm{C}^{18} \mathrm{O}(2-1)$. The HPBW of the telescope is $125^{\prime \prime}$ and $70^{\prime \prime}$ at $220 \mathrm{GHz}$ and $345 \mathrm{GHz}$, respectively. The telescope has a dual-channel-SIS receiver $(230 / 345 \mathrm{GHz})$ with typical receiver temperatures in the range $100-180 \mathrm{~K}$ (SSB). Three different acousto-optical spectrometers were used: a Medium Resolution AOS with $300 \mathrm{MHz}$ bandwidth and a resolution of $360 \mathrm{kHz}$ (August), a High Resolution AOS with $59 \mathrm{MHz}$ bandwidth and $53 \mathrm{kHz}$ resolution, and a Variable Resolution AOS fixed at a bandwidth of $655 \mathrm{MHz}$ and a resolution of $550 \mathrm{kHz}$ (October). Therefore, the velocity resolutions for both frequency ranges were similar, about $0.4 \mathrm{~km} \mathrm{~s}^{-1}$. The pointing was checked regularly and it is estimated to be about $10^{\prime \prime} \mathrm{rms}$ in Az and El. The observations were made in the position switching mode.

\footnotetext{
${ }^{2}$ downloaded from the SkyView, Web version: v4.1, http://skyview.gsfc.nasa.gov/
} 

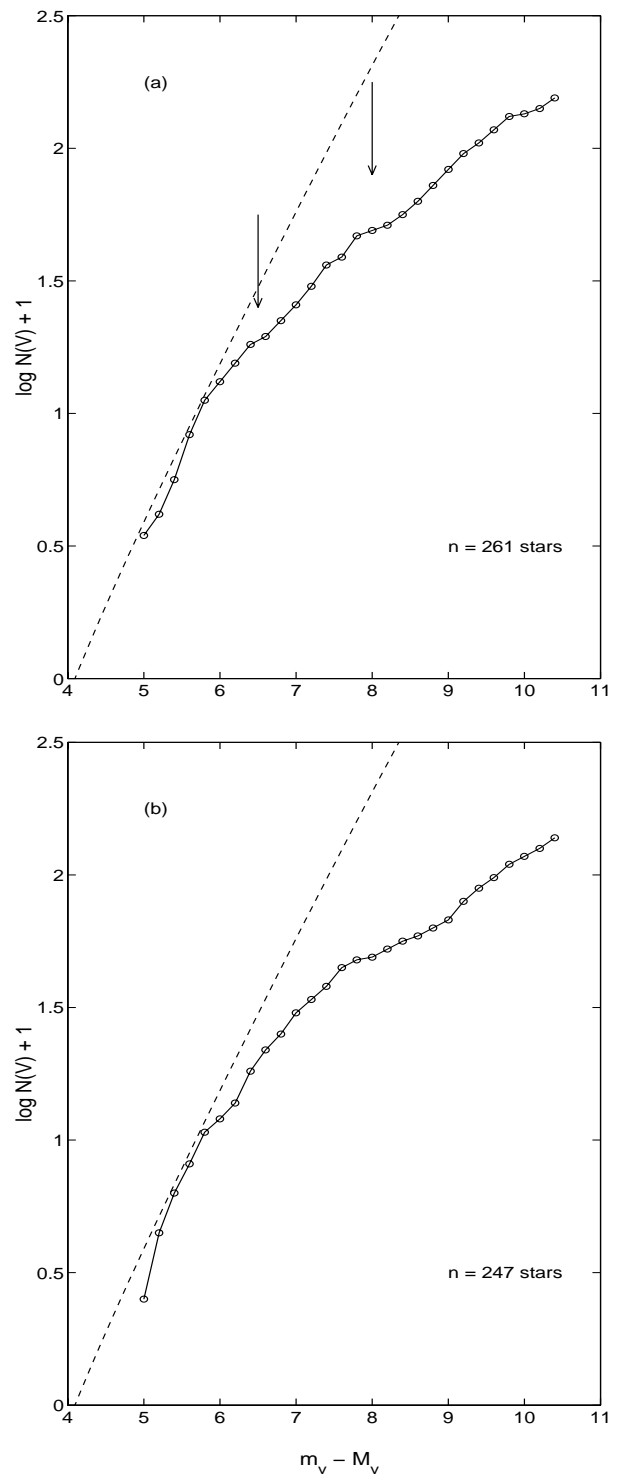

Fig. 1. Wolf diagrams for the L 1274 Schmidt field: a) logarithm of the cumulative number of $\mathrm{A}$ and $\mathrm{F}$ type stars per square degree as a function of the distance modulus in a $3^{\circ} \times 3^{\circ}$ box centred at $\alpha_{1950.0}=23^{\mathrm{h}} 50^{\mathrm{m}}, \delta_{1950.0}=+70^{\circ} 52^{\prime} .5$. The dashed line is the extinction free function calculated from the Galaxy model by Wainscoat et al. (1992) for the Galactic coordinates $\ell=118^{\circ}, b=9^{\circ}$. Two absorption layers are indicated. b) the same as in a) but for the area outside the $3^{\circ} \times 3^{\circ}$ box. See the text below

\section{Results}

\subsection{Distance}

The cloud has a small area compared to the field of view of the Schmidt telescope. We decided, therefore, to examine progressively larger regions, centred on the L 1274 $\mathrm{CO}$ cloud $\left(\alpha_{1950.0}=23^{\mathrm{h}} 50^{\mathrm{m}}, \delta_{1950.0}=+70^{\circ} 53^{\prime}\right)$, and investigate whether absorption layers detected in a smaller region persist in larger ones. Boxes of $1^{\circ} \times 1^{\circ}$ and $2^{\circ} \times 2^{\circ}$ did not have enough stars for reliable statistics, but they indicated the existence of two absorption layers. In Fig. 1 we present Wolf diagrams of a $3^{\circ} \times 3^{\circ}$ area (centred on the CO cloud) with 261 stars (a) and of the rest of the Schmidt's field of view, with 247 stars (b). To construct a reference extinction - free curve we used parameters given in Wainscoat et al. (1992).

Both Wolf-curves show two layers of obscuring material. The difference between the two diagrams is that the nearest absorption layer is more shallow in the latter field, (b), probably because here we see the more transparent parts of that layer. This indicates that the L 1274 cloud is located at the smallest distance. At a distance modulus of 6.2 (corresponding to a distance of $\sim 170 \mathrm{pc}$ ) the difference between the model curve and the observed one starts to be statistically significant (when defined by the statistical error of the star counts, $N^{1 / 2}$ ). We consider this as a lower limit to the distance modulus of L 1274 since errors in the spectral classification and dispersion in the absolute magnitudes of the stars smooth the effects of absorption layers in the Wolf diagram. In the ideal case, an upper limit of the distance modulus (i.e. the far side of the cloud) is defined by the point where the observed curve again turns parallel to the model curve; the closest match occurs at about $6.7^{\mathrm{mag}}$ (i.e. $220 \mathrm{pc}$ ). We thus assign a distance modulus of $6.5^{\mathrm{mag}}$ to L 1274 , corresponding to a distance of $200 \mathrm{pc}$, with an uncertainty of about $30 \mathrm{pc}$. The average absorption of this layer is $0.7^{\mathrm{mag}}$ (in a $3^{\circ} \times 3^{\circ}$ area).

The more remote absorption layer might be part of the $400 \mathrm{pc}$ component layer seen in the Cepheus region towards lower Galactic latitudes (Kun 1998). Close to L 1274, and included in our Schmidt-plate, lies another cloud which in the FIR has a diameter of $\sim 10^{\prime}$. We have labeled this cloud "L $1274 \mathrm{~N}$ ". It is bright both at 60 and $100 \mu \mathrm{m}$, with the peak IR brightness position at $\Delta \ell=+36^{\prime}, \Delta b=+62^{\prime}$ relative to the $(0,0)$ position of our $\mathrm{CO}$ map. ${ }^{12} \mathrm{CO}(1-0)$ test measurements (Onsala) show emission at $v_{\mathrm{LSR}}=-10.9 \mathrm{~km} \mathrm{~s}^{-1}$. The observed velocity suggests that this cloud may belong to the more remote absorption layer seen in our Wolf diagrams. The average absorption is about $1.3^{\mathrm{mag}}$.

\subsection{Dust distribution}

The L 1274 cloud has moderate FIR emission at $100 \mu \mathrm{m}$, but lacks significant emission at $60 \mu \mathrm{m}$, a property indicative of dark clouds (Laureijs et al. 1991, Abergel et al. 1994). Laureijs et al. (1991) modeled the FIR emission of the dark clouds complex containing L 134, L 183 and L 1780; assuming a $\lambda^{-1}$ dust emissivity law the absence of $60 \mu \mathrm{m}$ emission yielded an upper limit of about $15 \mathrm{~K}$.

The $100 \mu \mathrm{m}$ excess emission is defined as $\Delta I_{100}=$ $I_{100}-I_{60} / \Theta$, where $\Theta$ is the ratio $I_{60} / I_{100}$ in the outer diffuse regions (Laureijs et al. 1991; Boulanger et al. 1998). We have used their ratio of $\Theta=0.2$, since in the $\mathrm{L} 1274$ region we could not derive it accurately due to the weak FIR emission. $\Delta I_{100}$ for L 1274 is spatially very well correlated with the ${ }^{13} \mathrm{CO}$ emission, as shown in Fig. 2a. There is also 

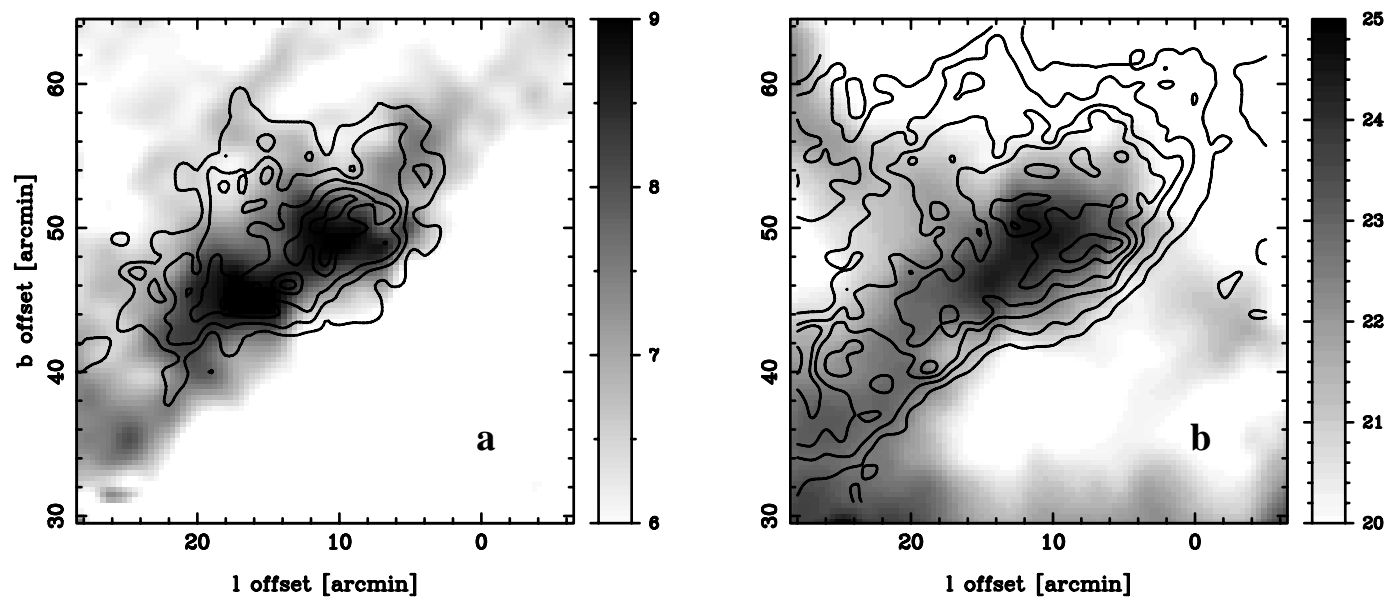

Fig. 2. a) The $100 \mu \mathrm{m}$ excess emission (greyscale) towards L 1274 overlayed with the ${ }^{13} \mathrm{CO}(1-0)$ contours. b) The $100 \mu \mathrm{m}$ emission (greyscale) overlayed with the ${ }^{12} \mathrm{CO}(2-1)$ contours. Greyscale is in $\mathrm{MJy} \mathrm{sr}^{-1}$ units. The contour plots show the total integrated CO emission from $20 \%$ level in steps of $20 \%$

a good correlation between the ${ }^{12} \mathrm{CO}$ and the $100 \mu \mathrm{m}$ emission, as seen in Fig. 2b. A comparative analysis of the Far $\mathrm{IR},{ }^{13} \mathrm{CO}$ and ${ }^{12} \mathrm{CO}$ data for dark cloud regions (see, e.g., Abergel et al. 1994) shows that the $100 \mu \mathrm{m}$ excess emission and the ${ }^{13} \mathrm{CO}$ emission have very similar distributions. Since the definition of $\Delta I_{100}$ should include a subtraction of the diffuse component of the cloud, the $100 \mu \mathrm{m}$ excess emission seems to be tightly correlated with molecular gas of densities $>10^{3} \mathrm{~cm}^{-3}$ (Laureijs et al. 1991).

Four point sources were found in this region. As their flux quality was poor at most wavelengths, the IRAS detector scans were examined using the Groningen Image Processing Software System, GIPSY (Assendorp et al. 1995). We classified them according to their infrared colours. Two sources, IRAS 23536+7043 and IRAS $23500+7100$, appear to be late type giants and agree within $5^{\prime \prime}$ with the positions of faint $\left(m_{\mathrm{pg}}<13^{\mathrm{mag}}\right)$ stars on the POSS plates. IRAS 23491+7053 coincides optically with a very faint, diffuse object and is probably a galaxy. The only source that lacks an optical counterpart (within $20^{\prime \prime}$ ) is IRAS $23505+7047$. The derived infrared colours indicate a cold source and our $\mathrm{CO}$-observations suggest that this source is associated with L 1274 .

\subsection{CO distributions and kinematics}

The CO channel maps in Figs. 3 and 4 show two distinct structures: the "main body" of the cloud emitting within the velocity range -5 to $-2.5 \mathrm{~km} \mathrm{~s}^{-1}$ and the "ridge" seen between -2 and $0 \mathrm{~km} \mathrm{~s}^{-1}$. Whether the two structures are unrelated or have a common origin is not clear. However, the morphology favours the second view: the channel maps in Fig. 3 show that the ridge follows closely the edge of the main body and that the main body seems to turn gradually into the ridge, spatially as well as kinematically. This is also apparent in Fig. 4 which shows similar distributions for all the three CO transitions observed. Those of
${ }^{12} \mathrm{CO}(1-0)$ and $(2-1)$ are remarkably similar with approximately the same extents. At a distance of $200 \mathrm{pc}$ the linear extent of the main body is 1.5 to $2 \mathrm{pc}$ as defined by the lowest $(10 \%)$ contours in Fig. 4. In ${ }^{13} \mathrm{CO}(1-0)$ the main body is well defined while the ridge has barely any emission, indicating that the ridge consists of more diffuse gas. The ${ }^{12} \mathrm{CO}(1-0) /{ }^{13} \mathrm{CO}(1-0)$ integrated intensity ratios are smaller than 5 over a significant part of the main body. In the ridge typical ratios are 10 or higher with the exception of the SE part where ratios are similar to those in the main body. The highest ratios in the ridge are found in an area surrounding IRAS 23505+7047 (indicated in Fig. 3).

Similar to the ${ }^{12} \mathrm{CO} /{ }^{13} \mathrm{CO}$ isotopic ratio, we see only small variations of the ${ }^{12} \mathrm{CO}(2-1) /{ }^{12} \mathrm{CO}(1-0)$ integrated intensity ratios in the main body. With the $\mathrm{CO}(1-0)$ data convolved to the same beam size as the $(2-1)$, this ratio is close to 0.7 when the beam efficiencies given in Table 1 are applied. Roughly the same ratio is found along a line through the centre of the ridge with a possible exception of the area around the IRAS source where it drops to $0.5-$ 0.6. At the edges of the ridge this ratio decreases quickly outwards.

We have investigated the kinematics of the gas with a number of position - velocity maps across the region. We see no other prominent velocity components than those associated with the main body and the ridge. The former shows no discernible velocity gradients. In the ridge, on the other hand, there is such a tendency with a velocity closer to that of the main body in the SE part. The difference is about $0.5 \mathrm{~km} \mathrm{~s}^{-1}$ between the SE $\left(v \sim-2 \mathrm{~km} \mathrm{~s}^{-1}\right)$ and the NW $\left(-1.5 \mathrm{~km} \mathrm{~s}^{-1}\right)$ regions (see Fig. 3$)$. It thus seems that the densest part (as indicated by low ${ }^{12} \mathrm{CO}(1-0) /{ }^{13} \mathrm{CO}(1-$ 0 ) ratios) of the ridge is closer to the main body, at least in velocity but, of course, not necessarily in space.

Finally, the line width of the main body emission is typically $1 \mathrm{~km} \mathrm{~s}^{-1}$ in the ${ }^{12} \mathrm{CO}$ lines while that of the ridge seems to be somewhat larger, $1.5 \mathrm{~km} \mathrm{~s}^{-1}$. The latter value is, however, uncertain due to the weakness of the line in 


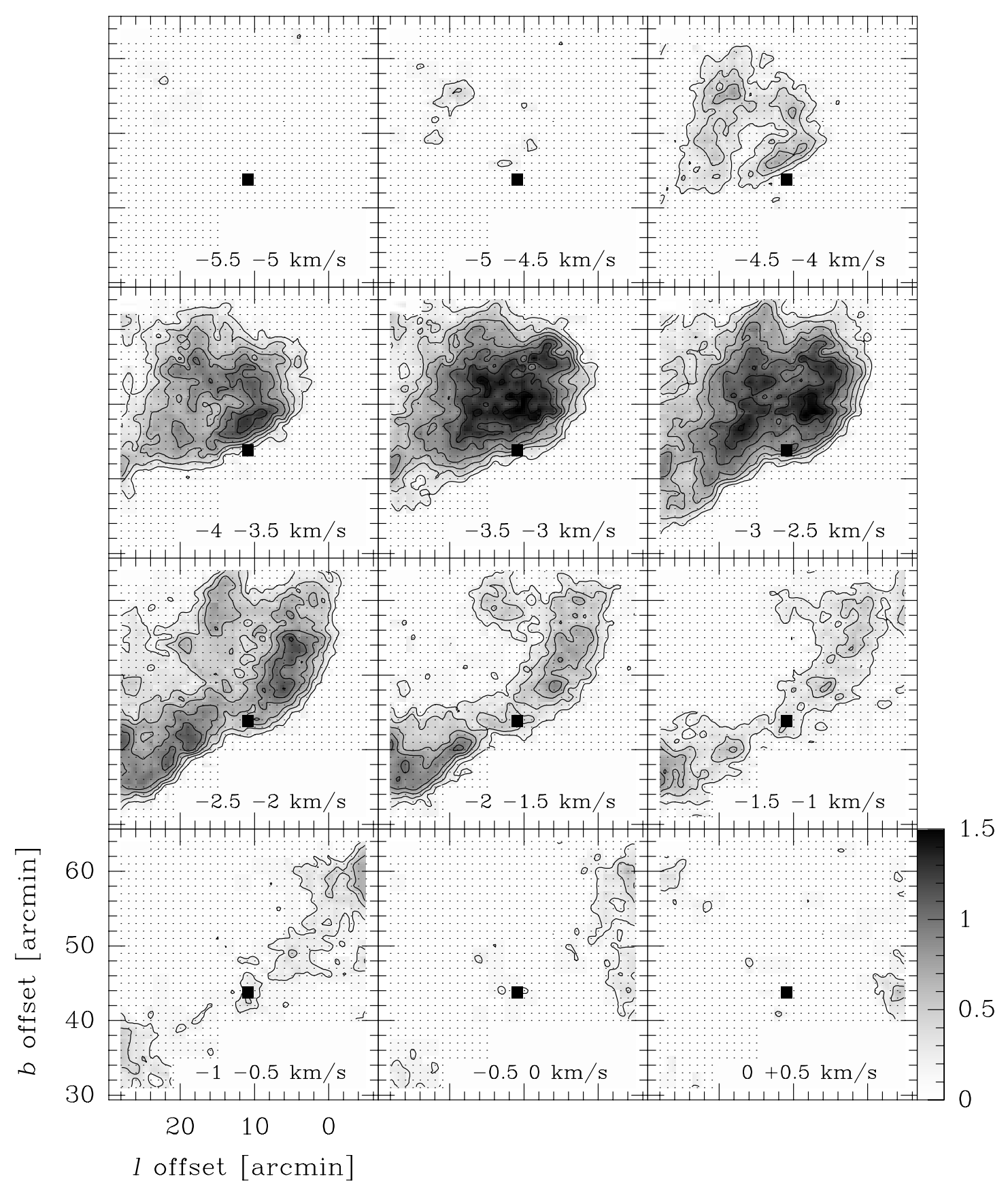

Fig. 3. ${ }^{12} \mathrm{CO}(2-1)$ channel maps. The velocity ranges are indicated in the lower right corner of each panel. The observed positions are indicated by dots. The intensity scale is in $T_{\mathrm{A}}^{*} \mathrm{~d} v\left[\mathrm{~K} \mathrm{~km} \mathrm{~s}^{-1}\right]$. Offsets are relative to $\ell=118.00, b=8^{\circ} .00$. The square marks the position of the IRAS PS classified as a cold clump

combination with blending by the stronger main body emission. Global profiles of the observed region indicate a larger difference between the two components, about a factor of 2 , likely due to the possible velocity gradient along the ridge.

\subsection{Physical state of the gas}

To estimate cloud conditions we have made smaller maps of the central part of $\mathrm{L} 1274$ in the $3-2\left({ }^{12} \mathrm{CO},{ }^{13} \mathrm{CO}\right)$ and $2-1\left({ }^{13} \mathrm{CO}, \mathrm{C}^{18} \mathrm{O}\right)$ transitions in addition to the full maps in ${ }^{12} \mathrm{CO}(1-0),(2-1)$ and ${ }^{13} \mathrm{CO}(1-0)$. At the position $\Delta \ell=+9^{\prime}, \Delta b=+50^{\prime}$, we have convolved the data to the KOSMA CO $(2-1)$ beam response $\left(\mathrm{HPBW} \cong 2^{\prime}\right)$. The convolved spectra are shown in Fig. 5 in the $T_{\mathrm{A}}^{*}$ intensity scale. To define appropriate beam efficiencies is not trivial. In the Onsala case the beam efficiency should be in the range 0.5 to 0.8 , where the former applies to a source covering the main-beam, while the latter corresponds to the efficiency measured on the Moon. Due to the large extent of the emission, we apply 0.8 for Onsala in 

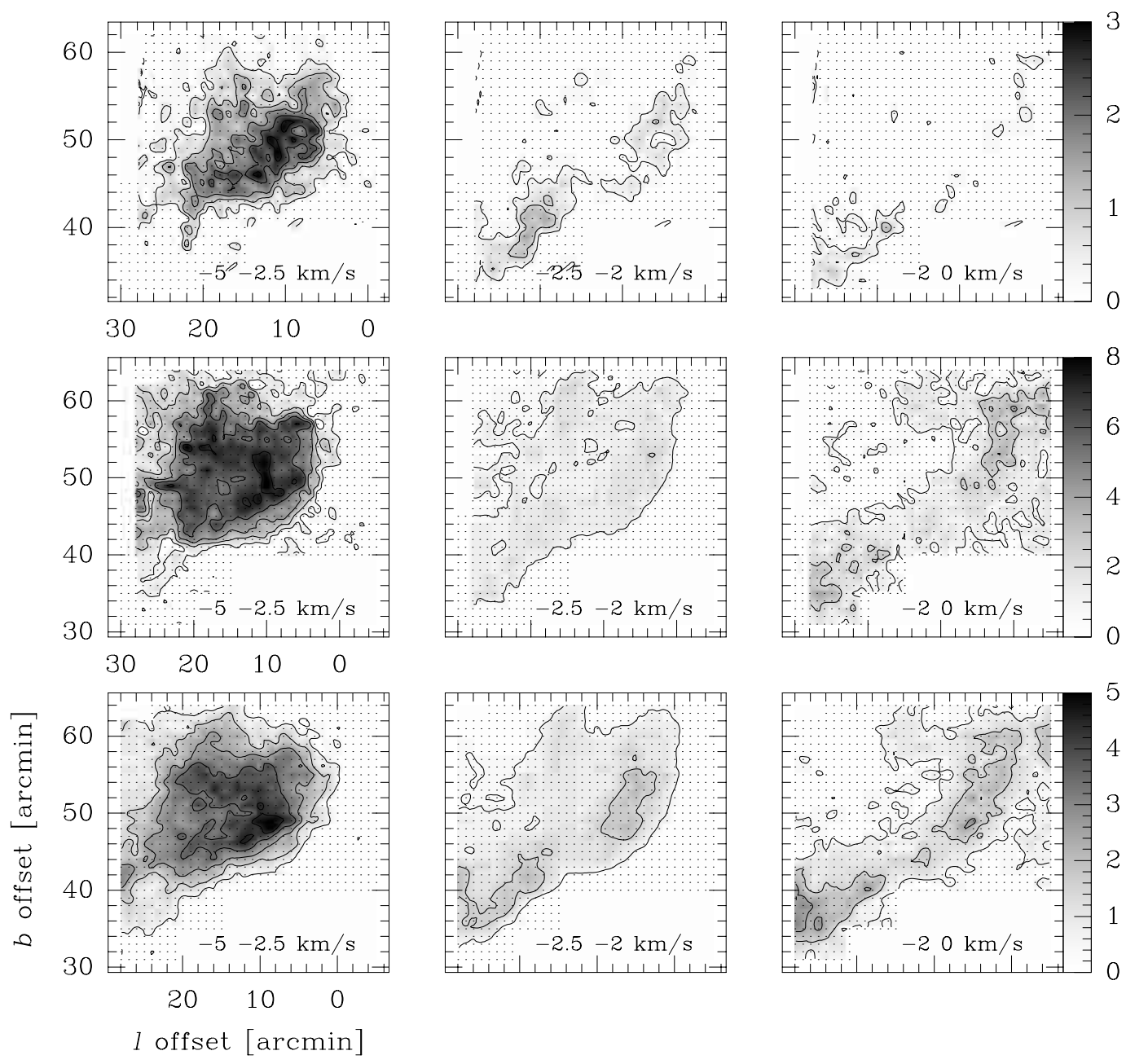

Fig. 4. CO channel maps. The velocity ranges are indicated in the lower right corner of each panel. First row: ${ }^{13} \mathrm{CO}(1-0)$ maps, contours from 0.3 by 0.6 to $3.0 \mathrm{~K} \mathrm{~km} \mathrm{~s}^{-1}$; second row: ${ }^{12} \mathrm{CO}(1-0)$ maps, contours from 0.8 by 1.6 to $8.0 \mathrm{~K} \mathrm{~km} \mathrm{~s}^{-1}$; third row: ${ }^{12} \mathrm{CO}(2-1)$ maps, contours from 0.5 by 1.0 to $5.0 \mathrm{~K} \mathrm{~km} \mathrm{~s}^{-1}$. The observed positions are indicated by dots. The intensity scale is in $T_{\mathrm{A}}^{*} \mathrm{~d} v\left[\mathrm{~K} \mathrm{~km} \mathrm{~s}^{-1}\right]$. Offsets are in arcminutes relative to $\ell=118^{\circ} .00, b=8^{\circ} .00$

Table 1. Beam efficiency corrected temperatures in the central part $\left(\Delta \ell=+9^{\prime}, \Delta b=+50^{\prime}\right)$ of L 1274. All intensities refer to the KOSMA ${ }^{12} \mathrm{CO}(2-1)$ beam response. Adopted beam efficiencies: $0.8,0.7$ and 0.6 for 110,220 and $345 \mathrm{GHz}$, respectively

\begin{tabular}{ccrr}
\hline Molecule & Transition & $T[\mathrm{~K}]$ & $I\left[\mathrm{~K} \mathrm{~km} \mathrm{~s}^{-1}\right]$ \\
\hline${ }^{12} \mathrm{CO}$ & $1-0$ & 7.1 & 11.8 \\
& $2-1$ & 4.3 & 8.2 \\
& $3-2$ & 4.0 & 7.7 \\
\hline${ }^{13} \mathrm{CO}$ & $1-0$ & 3.1 & 3.1 \\
& $2-1$ & 2.3 & 2.5 \\
& $3-2$ & 0.63 & 0.75 \\
\hline $\mathrm{C}^{18} \mathrm{O}$ & $1-0$ & 0.44 & 0.33 \\
& $2-1$ & 0.33 & 0.32 \\
\hline
\end{tabular}

the 110-115 GHz range. The KOSMA efficiencies are even less certain due to panel replacements and limited beam efficiency information in the period of our observations. We have used 0.7 and 0.6 at 230 and $345 \mathrm{GHz}$, respectively. Table 1 gives the corrected peak temperatures and integrated line intensities.

In Table 2 we present the results of an LTE and a non-LTE analysis of the data given in Table 1. The latter analysis uses a statistical-equilibrium excitation and radiative transfer (mean escape probability approximation) code assuming a spherical cloud with constant temperature and density (a model developed by J. H. Black and described in e.g. Jansen et al. 1994). Below we refer to this as the MEP analysis. In the LTE assumption we have used the following relations: 
Table 2. LTE and MEP solutions for the data in Table 1. $R_{n k}$ are the ${ }^{12} \mathrm{CO}$ integrated intensity ratios of the transitions with upper levels $n$ and $k$. The listed uncertainties in the derived parameters correspond to $\chi^{2}$-numbers twice those of the best fits

\begin{tabular}{lccccccccc}
\hline & $T\left({ }^{12} \mathrm{CO}(1-0)\right)$ & \multicolumn{3}{c}{$I\left({ }^{12} \mathrm{CO}\right) / I\left({ }^{13} \mathrm{CO}\right)$} & $R_{21}$ & $R_{31}$ & \multicolumn{2}{c}{$N\left({ }^{13} \mathrm{CO}\right) / N\left(\mathrm{C}^{18} \mathrm{O}\right)$} & $N\left({ }^{12} \mathrm{CO}\right)^{1)}$ \\
& {$[\mathrm{K}]$} & $1-0$ & $2-1$ & $3-2$ & & & $1-0$ & $2-1$ & {$\left[10^{17} \mathrm{~cm}^{-2}\right]$} \\
\hline observed & $7.1 \pm 0.5$ & $3.8 \pm 0.3$ & $3.3 \pm 0.4$ & $10.0 \pm 2$ & 0.70 & 0.65 & $9.4^{2)}$ & $7.8^{2)}$ & \\
$\mathrm{LTE}^{3)}$ & 6.5 & 3.7 & 2.9 & 6.0 & 0.78 & 0.57 & 10.9 & 9.6 & $4 \pm 1$ \\
$\mathrm{MEP}^{4)}$ & 7.5 & 3.8 & 3.8 & 10.0 & 0.80 & 0.54 & 10.4 & 8.5 & $2.5 \pm 0.5$ \\
\hline
\end{tabular}

1) $N\left({ }^{12} \mathrm{CO}\right) / N\left({ }^{13} \mathrm{CO}\right)=75$ assumed.

2) Integrated intensity ratio.

3) $T_{\mathrm{ex}}=10 \pm 2 \mathrm{~K} ;\left\langle\tau_{13}\right\rangle=0.32 ; 0.43 ; 0.19$ for the (1-0), $(2-1)$ and (3-2) transitions, respectively.

4) $T_{\mathrm{K}}=11 \pm 1 \mathrm{~K} ; n_{\mathrm{H}_{2}}=5_{-2}^{+5} 10^{3} \mathrm{~cm}^{-3}$.

where $\eta$ is the beam efficiency, $\tau$ the optical depth, $T_{\mathrm{ex}}$ is the excitation temperature, $T_{\mathrm{bg}}$ is the cosmic background radiation $(2.73 \mathrm{~K})$ and

$J(T)=\frac{h \nu}{k}\left(\mathrm{e}^{\frac{h \nu}{k T}}-1\right)^{-1}$

where $h$ is the Planck and $k$ is the Boltzmann constant, and $\nu$ is the frequency of the transition. For the ${ }^{13} \mathrm{CO}$ column density, $N_{13}$, we have used the LTE formula for linear rotors (see, e.g., Heikkilä et al. 1999) corrected by the optical depth

$N_{13}\left[\mathrm{~cm}^{-2}\right]=4.7210^{13} \frac{T_{\mathrm{ex}} \mathrm{e}^{5.3 / T_{e x}}}{1-\frac{J\left(T_{\mathrm{bg}}\right)}{J\left(T_{\mathrm{ex}}\right)}} \frac{\left\langle\tau_{13}\right\rangle}{1-\mathrm{e}^{-\left\langle\tau_{13}\right\rangle}} I_{13}$

$I_{13}$ is the integrated antenna temperature $\left[\mathrm{K} \mathrm{km} \mathrm{s}^{-1}\right] \mathrm{di}^{-}$ vided by the beam efficiency and $\left\langle\tau_{13}\right\rangle$ is the optical depth in the ${ }^{13} \mathrm{CO}$ line, determined from the ratio of the observed ${ }^{12} \mathrm{CO}$ and ${ }^{13} \mathrm{CO}$ integrated intensities. In this sense, $\left\langle\tau_{13}\right\rangle$ is a weighted average over the line profiles and thus more appropriate than the peak value when calculating column densities. To derive $\left\langle\tau_{13}\right\rangle$ and $T_{\text {ex }}$, we assume that the excitation temperatures are equal for ${ }^{12} \mathrm{CO}$ and ${ }^{13} \mathrm{CO}$.

Since the applied beam efficiencies suffer from relatively large uncertainties, the model solutions presented in Table 2 are defined by the ${ }^{12} \mathrm{CO} /{ }^{13} \mathrm{CO}$ integrated intensity ratios which are largely independent of the efficiencies. However, to better constrain the solutions, we have also used the ${ }^{12} \mathrm{CO}(1-0)$ line temperature and assumed complete beam filling. These parameters were fitted to a grid of models with different $T_{\mathrm{ex}}$ (LTE) or $T_{\mathrm{kin}}, n_{\mathrm{H}_{2}}$ and $N$ (CO) (MEP) by minimizing $\chi^{2}$ under the assumption of a ${ }^{12} \mathrm{C} /{ }^{13} \mathrm{C}$ abundance ratio of 75 ; the rms errors assigned to the data are given in Table 2 . We have also indicated the observed and modeled ${ }^{12} \mathrm{CO}$ intensity ratios of the different transitions, ratios which suffer from uncertainties in the beam efficiencies and the convolutions. Indicated are, also, the total column density ratios of ${ }^{13} \mathrm{CO}$ and $\mathrm{C}^{18} \mathrm{O}$ as derived from the $(1-0)$ and $(2-1)$ transitions, assuming the parameter values derived in the $\chi^{2}$ fits. They are all close to the expected ratio $\sim 8$, i.e. with ${ }^{12} \mathrm{C} /{ }^{13} \mathrm{C}$ and ${ }^{16} \mathrm{O} /{ }^{18} \mathrm{O}$

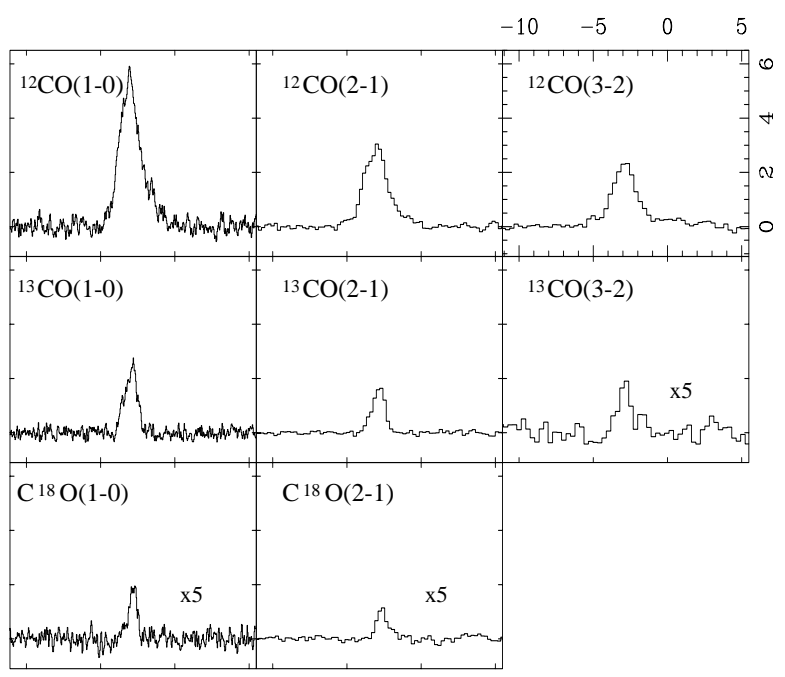

Fig. 5. $T_{\mathrm{A}}^{*}$ scale spectra at $\Delta \ell=+9^{\prime}, \Delta b=+50^{\prime}$ convolved to mimic the KOSMA ${ }^{12} \mathrm{CO}(2-1)$ beam response. Note that the ${ }^{13} \mathrm{CO}(3-2)$ and $\mathrm{C}^{18} \mathrm{O}$ spectra are multiplied by a factor of $5 \mathrm{in}$ intensity
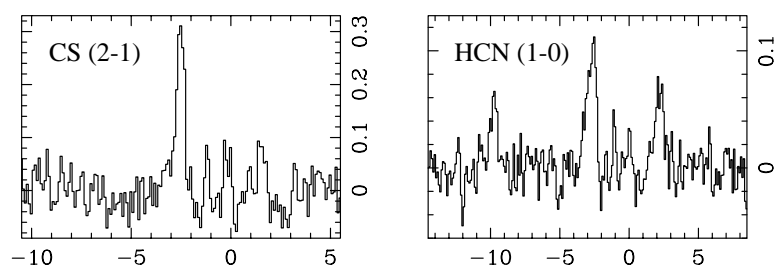

Fig. 6. CS and HCN $T_{\mathrm{A}}^{*}$ scale spectra at $\Delta \ell=+9^{\prime}, \Delta b=+50^{\prime}$

abundance ratios of about 75 and 560, respectively, in the local ISM (Wilson \& Rood 1994).

The MEP - solution for $\mathrm{CO}$ gives a $\mathrm{H}_{2}$ - density of about $510^{3} \mathrm{~cm}^{-3}$. However, we have also detected some higher density tracers (CS and HCN; see Fig. 6), indicating densities $>10^{4} \mathrm{~cm}^{-3}$. For example, the MEP solution for HCN, assuming $T_{\text {kin }}=10 \mathrm{~K}$, gives a density of $510^{4} \mathrm{~cm}^{-3}$ and a column density of $10^{12} \mathrm{~cm}^{-2}$.

In Table 3 we present mass estimates resulting from different methods. A crucial parameter is the distance and 
we have used $200 \mathrm{pc}$ obtained from our optical measurements. However, we have also applied the size-line width relation for molecular clouds as defined by ${ }^{12} \mathrm{CO}(1-0)$ data (Solomon et al. 1987) to get independent estimates. We have used this relation only to the "main body" of the $\mathrm{CO}$ emission. The dispersion in the size-line width relation by Solomon et al. (1987) corresponds to $50 \mathrm{pc}$ at a distance of $200 \mathrm{pc}$. If we apply this uncertainty, all estimates agree within the errors with the adopted distance of 200 pc.

The virial mass estimates are based on the relation (Johansson et al. 1998)

$M_{\mathrm{vir}}=150 d \Delta v^{2}\left[M_{\odot}\right]$

where $d[\mathrm{pc}]$ is the diameter of the cloud as defined by the half intensity contour of the CO emission and $\Delta v\left[\mathrm{~km} \mathrm{~s}^{-1}\right]$ is the full halfwidth of the global line profile of the cloud. More specifically, the diameter is determined by fitting a two-dimensional Gaussian function to the observed intensity distribution and use the resulting harmonic mean of the major and minor axis extents. For further details of this method to derive cloud parameters, see Johansson et al. (1998).

Below we focus on mass and density estimates for the main body, although some more uncertain estimates for the weaker ridge emission are given, too. The ridge may well be a separate cloud or, if associated with the main body, the result of an external or internal activity. Thus, e.g. mass estimates from the virial theorem are probably less meaningful in the latter case, further emphasised by the fact that the ridge emission seems to extend beyond the mapped region. To minimize effects of the ridge component we derived the main body parameters in a smaller area than the one mapped (see Fig. 4): offsets larger than $40^{\prime}$ and $0^{\prime}$ in latitude and longitude, respectively. The global profiles $\left({ }^{12} \mathrm{CO},{ }^{13} \mathrm{CO}\right)$ of this area showed weak emission from the ridge in addition to that of the main body. A two component Gaussian fit of the global line profile was applied to determine $\mathrm{CO}$-luminosities and velocity widths associated with the main body of the cloud. To define the spatial extent we used the distribution of the emission integrated over the velocity range -5.5 to $-2.5 \mathrm{~km} \mathrm{~s}^{-1}$.

With a $\mathrm{CO}-$ to $-\mathrm{H}_{2}$ conversion factor of $1.910^{20} \mathrm{~cm}^{-2}$ $\left(\mathrm{K} \mathrm{km} \mathrm{s}^{-1}\right)^{-1}$ (Strong \& Mattox 1996) and a correction for helium of $36 \%$ by mass, the total gas mass estimated from the $\mathrm{CO}(1-0)$ data is

$M_{x}=4.13 L_{\mathrm{co}}\left[M_{\odot}\right]$

where $L_{\mathrm{co}}$ is the $\mathrm{CO}$-luminosity in units of $\mathrm{K} \mathrm{km} \mathrm{s}^{-1} \mathrm{pc}^{2}$.

A third estimate, from the ${ }^{13} \mathrm{CO}$ emission, uses the ratio $\left[\mathrm{H}_{2}\right] /\left[{ }^{13} \mathrm{CO}\right]=4.810^{5}$ (Dickman \& Clemens 1983).

As seen in Table 3 the virial masses derived are a factor of 3-17 higher than other mass estimates. A similar difference between virial masses and masses derived from the $\mathrm{CO}-$ to $-\mathrm{H}_{2}$ conversion factor is typical for high-latitude molecular clouds (HLCs), a fact explained in terms of
Table 3. Estimates of distance and mass of the main body in $\mathrm{L} 1274$. The distance derived from the Wolf diagram is $200 \mathrm{pc}$, which we have adopted for all mass estimates. At this distance, $10^{\prime}$ corresponds to a linear size of $0.58 \mathrm{pc}$. The error estimates are formal $1 \sigma$ uncertainties. For the virial masses the uncertainties in the diameters and linewidths contribute roughly equally. In the other mass estimates, the absolute intensity scale uncertainty is the dominant factor

\begin{tabular}{lrccc}
\hline & & $\mathrm{CO}(1-0)$ & ${ }^{13} \mathrm{CO}(1-0)$ & $\mathrm{CO}(2-1)$ \\
\hline$\Delta v$ & {$\left[\mathrm{~km} \mathrm{~s}^{-1}\right]$} & 1.51 & 1.12 & 1.65 \\
$d^{1)}$ & {$[\operatorname{arcmin}]$} & 18.6 & 12.0 & 17.7 \\
$r^{2)}$ & {$[\operatorname{arcmin}]$} & 14 & 10 & 13 \\
$D^{3)}$ & {$[\mathrm{pc}]$} & 190 & 160 & 230 \\
$M_{\text {vir }}$ & {$\left[M_{\odot}\right]$} & $370 \pm 30$ & $130 \pm 8$ & $420 \pm 35$ \\
$M_{x}{ }^{4)}$ & {$\left[M_{\odot}\right]$} & $43 \pm 4$ & & \\
$M_{13}{ }^{5)}$ & {$\left[M_{\odot}\right]$} & & $25 \pm 2$ & \\
$n_{\mathrm{H}_{2}}{ }^{6)}$ & {$\left[\mathrm{cm}^{-3}\right]$} & 320 & 460 & \\
\hline
\end{tabular}

1) Diameter of the emission ( $50 \%$ contour).

2) Radius of the emission ( $10 \%$ contour).

3) Distance determined from the size-line width relation by Solomon et al. (1987).

4) Mass estimate using a $\mathrm{CO}-$ to $-\mathrm{H}_{2}$ conversion factor of $1.910^{20} \mathrm{~cm}^{-2}\left(\mathrm{~K} \mathrm{~km} \mathrm{~s}^{-1}\right)^{-1}$ (Strong \& Mattox 1996).

5) Mass estimate assuming $\left[\mathrm{H}_{2}\right] /\left[{ }^{13} \mathrm{CO}\right]=4.810^{5}$ (Dickman \& Clemens 1983).

6) Mean density in a volume of radius $r$.

deviations from gravitational virial equilibrium (see, e.g., Heithausen 1996). To compare the properties of L 1274 with HLCs we have also analysed our data in the same way as Heithausen and others. The significant difference is the determination of the cloud size: a radius, $r$, is defined by the area within the $2 \sigma$ detection limit contour. In our case this detection limit corresponds to about $10 \%$ of the peak emission. The derived radii and the corresponding line widths are given in Table 3 . When using the size-line width relation and the expression for the virial mass in the paper by Heithausen (1996), we find distances larger by about $20 \%$ compared with those from the Solomon et al. (1987) relation while the virial masses show a closer agreement (assuming a distance of $200 \mathrm{pc}$ ). Table 3 also gives the mean density of $\mathrm{H}_{2}$ within a volume of radius $r$. This number is at least an order of magnitude larger than typical for HLCs (Heithausen 1996), thus indicating that the main body has properties more similar to ordinary dark cloud cores. This is further emphasized by the volume filling factor of the molecular gas, here defined as the ratio of the mean density and the density obtained from the MEP solution of CO, about $10 \%$ for L 1274 and less than $1 \%$ for HLCs (Heithausen 1996).

We have also estimated the mass for the ridge component from the ${ }^{12} \mathrm{CO}$ and ${ }^{13} \mathrm{CO}$ luminosities in the same way as for the main body. We find 25 and $3.5 M_{\odot}$ from the $\mathrm{CO}-$ to $-\mathrm{H}_{2}$ conversion factor and the $\left[\mathrm{H}_{2}\right] /\left[{ }^{13} \mathrm{CO}\right]$ ratio, respectively. This large difference (although highly uncertain due to the weak ridge emission and blending by the 
main body component) in the two estimates might be a result of isotope selective photodissociation of the CO gas, provided that the density in the ridge is low. Support for low gas densities in the ridge comes from the weak $\mathrm{CO}$ emission itself, as well as high ${ }^{12} \mathrm{CO} /{ }^{13} \mathrm{CO}$ intensity ratios (a factor of 2 higher than in the main body). Thus, our data indicate that the ridge has different properties, notably more dilute gas, than the main body.

\section{Discussion}

\subsection{Uncertainties on the masses derived}

As noted in the previous section, the large virial masses relative to other mass estimates for the main body point to an object with properties similar to HLCs while the mean density is more in accordance with that of dark cloud cores. This mass difference can be removed by assigning a larger distance to the cloud, since the virial mass is proportional to the distance while the other mass estimates relate to the distance squared. Thus, a distance larger by a factor of $5\left({ }^{13} \mathrm{CO}\right.$ data) or about $9\left({ }^{12} \mathrm{CO}\right)$ would result in similar masses and a mean density closer to what is observed in HLCs. At such distances, however, the cloud would not be detectable by star counts. Another possibility is of course that we underestimate the mass derived from the ${ }^{12} \mathrm{CO}$ and ${ }^{13} \mathrm{CO}$ data by an order of magnitude. For example, Boulanger et al. (1998) find such deviations between masses calculated from the CO-to- $\mathrm{H}_{2}$ conversion factor and those derived from $100 \mu \mathrm{m}$ data, for some small clouds in the Chamaelon complex, although the two mass estimates agree satisfactorily in the region as a whole. They attribute the differencies to a CO deficient gas component, characterized by $100 \mu \mathrm{m}$ emission with warm IRAS color (excess of $60 \mu \mathrm{m}$ emission), while clouds with low $60 / 100 \mu \mathrm{m}$ flux ratios are better traced by CO. With respect to IRAS colors, L 1274 resembles the latter category, possibly indicating that our mass estimate from ${ }^{12} \mathrm{CO}$ suffers from an error less than an order of magnitude.

Our mass estimate from the ${ }^{13} \mathrm{CO}$ data relies on an $\left[\mathrm{H}_{2}\right] /\left[{ }^{13} \mathrm{CO}\right]$ ratio of $4.810^{5}$ (Dickman \& Clemens 1983), determined from a relatively large sample of local dark clouds (Dickman 1978) taken from the Lynds (1962) catalog. Their sizes, opacity classes and kinetic temperatures (Dickman 1978) are similar to those of L 1274. In this sense, the applied $\left[\mathrm{H}_{2}\right] /\left[{ }^{13} \mathrm{CO}\right]$ ratio should be quite reliable. Whether L 1274 is an extreme case in any other properties, like e.g. CO depletion or line saturation, is harder to decide. In an attempt to reveal such effects we have used the ratio of the $\mathrm{H}_{2}$ column densities as derived by the CO-to- $\mathrm{H}_{2}$ conversion factor (Strong \& Mattox 1996) and a $\left[\mathrm{C}^{18} \mathrm{O}\right] /\left[\mathrm{H}_{2}\right]$ ratio of $1.710^{-7}$ (Frerking et al. 1982):

$$
\frac{N\left(\mathrm{H}_{2}\right)_{\mathrm{CO}}}{N\left(\mathrm{H}_{2}\right)_{\mathrm{C}^{18} \mathrm{O}}}=\frac{1.910^{20} I_{\mathrm{CO}}}{5.910^{6} N_{18}}=0.3210^{14} \frac{I_{\mathrm{CO}}}{N_{18}}
$$

where $I_{\mathrm{CO}}$ is the velocity integrated ${ }^{12} \mathrm{CO}$ emission in $\mathrm{K} \mathrm{kms}^{-1}$ and $N_{18}$ is the $\mathrm{C}^{18} \mathrm{O}$ column density in $\mathrm{cm}^{-2}$.
Since this ratio, in most cases, involves an optically thick and thin line, it should be sensitive to physical and chemical (e.g. CO depletion or isotope selective processes) properties deviating from the "typical" case. To isolate a few cases (assuming that the CO isotopic ratios remain constant): i) if the $\mathrm{CO}$ gas is depleted, the $\mathrm{C}^{18} \mathrm{O}$ column density scales directly with the depletion while the saturated ${ }^{12} \mathrm{CO}$ emission $\left(\sim 1-\mathrm{e}^{-\tau_{\mathrm{CO}}}\right)$ is less affected, implying a ratio $>1$ and, (ii) if the ${ }^{12} \mathrm{CO}$ line is saturated to an extraordinary level, some fraction of the gas is missed in ${ }^{12} \mathrm{CO}$ giving a ratio $<1$. Applying formula (6) to the observed ${ }^{12} \mathrm{CO}(1-0)$ and $\mathrm{C}^{18} \mathrm{O}(1-0)$ data in the center position of L1274 (Table 1) we find a factor of 1.2, i.e. a close agreement between the two $\mathrm{H}_{2}$ column density estimates. To compare, we have chosen a well-studied dark cloud where several tracers indicate more extreme conditions: the dense, starless and collapsing core of L 1544 (see, e.g., Tafalla et al. 1998; Caselli et al. 1999). High density tracers, e.g. the CS(2-1) line, show the typical double-peaked line shapes indicative of matter infall (note that the CS(21) line observed towards L1274 lacks any tendency of a secondary peak). The ${ }^{12} \mathrm{CO}(1-0)$ line is extremely saturated with an estimated optical depth of $\sim 400$ (Tafalla et al. 1998); the corresponding optical depth in L 1274 is an order of magnitude lower (Table 2). Using the CO isotope data in Tafalla et al. (1998), formula (6) gives a ratio of 0.3 for $\mathrm{L} 1544$, at least an indication to question the mass estimates. It is interesting to note that Caselli et al. (1999) estimate, from $\mathrm{C}^{17} \mathrm{O}$ and $1.3 \mathrm{~mm}$ continuum observations, that the $\mathrm{CO}$ gas is depleted by a factor of 10 in $\mathrm{L} 1544$. If so, the $\mathrm{CO}-\mathrm{to}-\mathrm{H}_{2}$ conversion factor underestimates the $\mathrm{H}_{2}$ content by a factor of 30 in this core. Note, however, that this is likely not due to the suggested $\mathrm{CO}$ gas depletion but to the extreme saturation of the ${ }^{12} \mathrm{CO}$ line.

The discussion above indicates that the L1274 cloud has similar properties as the cloud sample for which the $\left[{ }^{13} \mathrm{CO}\right] /\left[\mathrm{H}_{2}\right]$ ratio is defined. Furthermore, all $\mathrm{CO}$ isotopes observed here give $\mathrm{H}_{2}$ column densities which agree closely, lending support to our mass estimates.

\subsection{Interpretation}

From the ${ }^{13} \mathrm{CO}$ survey of the Cep-Cas region Yonekura et al. (1997) also find high $M_{\text {vir }} / M_{13}$ ratios for clouds with $M_{13} \leq 100 M_{\odot}$. They estimated the external pressure in this region to $P_{\text {ext }} / k \sim 10^{5} \mathrm{~K} \mathrm{~cm}^{-3}$. This pressure is high enough to bind most of the clouds in their sample, except maybe the smallest ones. For L 1274, using the $M_{13}$ mass estimate, we find that the pressure required to bind the main body is $\sim 10^{4.5} \mathrm{~K} \mathrm{~cm}^{-3}$. This indicates that the main body might be pressure confined.

In any case, L 1274 and other clouds of similar properties are apparently not gravitationally bound. This suggests that they are, either, formed by another process than gravitation, or, disturbed by some internal or external process. Both cases might be explained by the passage of a 
shock front. In the former case, the shock front triggers the formation of a cloud, which, if gravitation does not take over, eventually evaporates. In the latter case, the shock front injects energy to the cloud, which may be seen, e.g., in form of increased turbulence. Support for a passage of a shock front comes from the channel maps in Figs. 3 and 4 which seem to indicate that the main body and the ridge component are related. In this scenario the ridge is an expanding low density feature that comes from the outer layers of the main body.

The question is, also, whether an external disturbance such as a shock wave is consistent with the observed IRAS colors. In the warm diffuse interstellar medium high speed shocks $\left(50<v_{\mathrm{S}}<200 \mathrm{~km} \mathrm{~s}^{-1}\right)$ change the grain size distribution towards smaller grains (e.g. Jones et al. 1996), which should decrease the $100 \mu \mathrm{m} / 60 \mu \mathrm{m}$ ratio. The situation is less clear for dense clouds and slow shocks. Draine et al. (1983) have studied MHD shocks in molecular clouds with preshock densities $n_{\mathrm{H}} \approx 10^{4}-10^{6} \mathrm{~cm}^{-3}$ and speeds in the range $5-50 \mathrm{~km} \mathrm{~s}^{-1}$. Magnetic fields enhance the sputtering of grain mantles, thus requiring shock waves of only $v_{\mathrm{s}} \geq 25 \mathrm{~km} \mathrm{~s}^{-1}$ for $\mathrm{H}_{2} \mathrm{O}$ ice mantles to be substantially eroded. Refractory grain cores, however, have binding energies an order of magnitude larger than $\mathrm{H}_{2} \mathrm{O}$ and will be almost unaffected by sputtering at these shock speeds (Draine et al. 1983). However, this model does not include grain-grain collisions which could change the size distribution of the grains. Observationally, one possible example is the $\rho$ Ophiuchi cloud where two distinct regions were found: a low-density, less depleted, predominantly atomic preshock gas and a predominantly molecular, more depleted postshock gas (Meyers et al. 1983). On the basis of UV and optical studies they inferred the presence of a shock wave with a velocity of about $10 \mathrm{~km} \mathrm{~s}^{-1}$ and traced the origin to $\mathrm{a} \sim 10^{6} \mathrm{yr}$ old SNR (Sancisi 1974). The age of the SNR is sufficient for grain enlargement to occur in the postshock gas (Meyers et al. 1983). They concluded that a low-velocity shock enhances grain growth in the postshock gas probably through some combination of accretion and coagulation, resulting in a grain distribution in which there is an excess of large grains and a deficiency of very small grains. Thus, a low velocity shock seems to be consistent with the observed $100 \mu \mathrm{m}$ excess emission in L 1274 .

The projected position of $\mathrm{L} 1274$ is in a region of two catalogued FIR loops: inside GIRL $116+10$ and at the periphery of GIRL $120+11$ (abbreviation "GIRL" stands for Galactic (F)IR Loop; Kiss et al. 2001, in prep.). Such loops are thought to trace dust and gas swept out by supernova explosions or strong stellar winds. If any of these loops has affected L 1274, the latter is the better candidate for geometrical reasons provided that the ridge is an extension of the main body. This is, however, very speculative. On the other hand, L 1274 seems to be in a rather violent area as indicated by soft $\mathrm{X}$-ray excess radiation, lack of $\mathrm{CO}$ emission and presence of radio-continuum loops. A scenario where a shock front from an old supernova explosion has already passed L 1274 is possible. For example, the centre and the estimated distance to the centre of Loop III (Berkhuijsen 1971, 1973) and the position and distance of L 1274 are consistent with passage of a SN - induced shock front during the last $10^{6}$ years. Assuming that the ridge has been swept away, the relative motions of the main body and the ridge in the radial direction are also consistent with this scenario (i.e. the ridge has gained some positive peculiar velocity relative to the main body).

Indications of internal disturbances are harder to find. Our data show that the main body is cold with no optical or IR signs of strong activity. Likewise, no large-scale motions like contraction or rotation are evident. There is a weak IR source at the edge of the main body (see Fig. 3), classified as a cold clump. However, the location of this source seems to correlate more with the ridge properties than those of the main body: the weakest $\mathrm{CO}$ emission (Fig. 4), the highest ${ }^{12} \mathrm{CO}(1-0) /{ }^{13} \mathrm{CO}(1-0)$ and the lowest ${ }^{12} \mathrm{CO}(2-1) /{ }^{12} \mathrm{CO}(1-0)$ ratios of the ridge are found close to the IR source (Sect. 3.3). The geometry of the ridge combined with the location of the IR source resembles a bipolar flow. As noted in Sect. 3.3 there is also, possibly, a small velocity gradient along the ridge, consistent with an almost face-on flow. Interpreted in this way, the ridge emission might then be the result of low mass star formation at the edge of the main body.

Anyway, the main conclusion of this discussion is that any internal activity (core collapse or star formation), which could explain the difference between our mass estimates, seems to be absent.

\section{Summary}

We present a multiwavelength study of L 1274, a dark cloud located in the weak CO emission area between the Cepheus and Cassiopeia molecular cloud complexes. Objective prism spectroscopy data were used to determine a distance of $200 \pm 30 \mathrm{pc}$ to the cloud.

ISSA plates were used to investigate the FIR emission. The L 1274 cloud has moderate $100 \mu \mathrm{m}$ and low $60 \mu \mathrm{m}$ emission. The $100 \mu \mathrm{m}$ excess emission (and the $100 \mu \mathrm{m}$ emission itself) is spatially well correlated with the core of the CO cloud.

We have used the OSO 20-m telescope to map the cloud in the ${ }^{12} \mathrm{CO}(1-0)$ and ${ }^{13} \mathrm{CO}(1-0)$ lines and the KOSMA 3-m telescope for a map in the ${ }^{12} \mathrm{CO}(2-1)$ line. A few positions were observed in the $\mathrm{C}^{18} \mathrm{O}(1-0), \mathrm{CS}(2-$ 1) and $\mathrm{HCN}(1-0)$ transitions (OSO) and the ${ }^{12} \mathrm{CO}(3-2)$, ${ }^{13} \mathrm{CO}(2-1)$ and $\mathrm{C}^{18} \mathrm{O}(2-1)$ transitions (KOSMA) to allow improved estimates of the gas properties. We find a kinetic temperature of $10-12 \mathrm{~K}$, an $\mathrm{H}_{2}$ density $>10^{4} \mathrm{~cm}^{-3}$ and a total column density of the ${ }^{12} \mathrm{CO}$ gas of $(2-5) 10^{17} \mathrm{~cm}^{-2}$ in the centre of the cloud.

The CO - maps show two velocity components, one designed as the "main body", a slightly elongated structure of $1.5-2 \mathrm{pc}$ in diameter, and a weaker filament component, which we designed as the "ridge". Our data indicate that the two components are related. 
For the main body we present mass estimates with different methods. Virial masses are higher by a factor of 3-17 than masses derived from the $\mathrm{CO}-$ to $-\mathrm{H}_{2}$ conversion factor and the ${ }^{13} \mathrm{CO}$ data using a standard $\left[\mathrm{H}_{2}\right] /\left[{ }^{13} \mathrm{CO}\right]$ ratio. We discuss the validity of these mass estimates but find no indications of extraordinary physical or chemical properties of the gas that would change significantly our estimates. Likewise, there are no obvious signs of an internal activity that could explain the difference between the virial masses and the $\mathrm{CO}$ mass estimates. We also discuss a possibility of a supernova induced shock front passage which, either, disturbed an existing cloud, or, triggered the formation of it. The ridge might be a consequence of such a passage.

Acknowledgements. Our sincere thanks are to Dr. Mária Kun and to the Konkoly Observatory for the telescope time, photoplates and hospitality. Prof. F. Sato (Nagoya) kindly provided an unpublished ${ }^{13} \mathrm{CO}$ map. Prof. J. Black (Onsala) made his code (revradex) available. We used Dr. P. Bergman's (Onsala) spectral line reduction package, XS.

Onsala Space Observatory is the Swedish National Facility for Radio Astronomy and is operated by Chalmers University of Technology, Göteborg, Sweden, with financial support from the Swedish Natural Science Research Council and the Swedish Board for Technical Development.

The KOSMA $3 \mathrm{~m}$-radio telescope at Gornergrat-Süd Observatory is operated by the University of Köln, and supported by the SFB-301, as well as by special funding from the Land NRW. The Observatory is administrated by the Internationale Stiftung Hochalpine Forschungsstationen Jungfraujoch und Gornergrat, Bern, Switzerland.

This research was partly supported by the OTKA grants F022566 and T-024027 and the JSPS-HAS Cooperative Science program No. 18.

\section{References}

Abergel, A., Boulanger, F., Mizuno, A., \& Fukui, Y. 1994, ApJ, 423, L59

Assendorp, R., Bontekoe, T. R., de Jonge, A. R. W., et al. 1995, A\&AS, 110, 395

Berkhuijsen, E. M. 1971, A\&A, 14, 252

Berkhuijsen, E. M. 1973, A\&A, 24, 143

Boulanger, F., Bronfman, L., Dame, T. M., \& Thaddeus, P. 1998, A\&A, 332, 273

Boulanger, F., \& Pérault, M. 1988, ApJ, 330, 964

Caselli, P., Walmsley, C. M., Tafalla, M., Dore, L., \& Myers, P. C. 1999, ApJ, 523, L165

Dame, T. M., Ungerechts, H., Cohen, R. S., et al. 1987, ApJ, 322,706

Dickman, R. L., \& Clemens, D. P. 1983, ApJ, 271, 143

Dickman, R. L. 1978, ApJS, 37, 407
Draine, B. T., Roberge, W. G., \& Dalgarno, A. 1983, ApJ, 264, 485

Frerking, M. A., Langer, W. D., \& Wilson, R. W. 1982, ApJ, 262,590

Grenier, I. A., Lebrun, F., Arnaud, M., et al. 1989, ApJ, 347, 231

Heikkilä, A., Johansson, L. E. B., \& Olofsson, H. 1999, A\&A, 344,817

Heithausen, A. 1996, A\&A, 314, 251

Jansen, D. J., van Dishoeck, E. F., \& Black, J. H. 1994, A\&A, 282,605

Johansson, L. E. B., Greve, A., Booth, R. S., et al. 1998, A\&A, 331,857

Jones, A. P., Tielens, A. G. G. M., \& Hollenbach, D. J. 1996, ApJ, 469, 740

Kiss, Cs., Tóth, L. V., Moór, A., Sato, F., Nikolić, S., \& Wouterloot, J. G. A. 2000, A\&A, 363, 755

Kramer, C., Degiacomi, C. G., Graf, U. U., et al. 1998, Proc. SPIE 3357, 711. Advanced Technology MMW, Radio and Terahertz Telescopes, ed. T. G. Phillips

Kun, M. 1998, ApJS, 115, 59

Kun, M., Obayashi, A., Sato, F. et al. 1994, A\&A, 292, 249

Lang, K. R. 1992, Astrophysical Data: Planets and Stars (Springer-Verlag New York, Inc.), 144

Laureijs, R. J., Clark, F. O., \& Prusti, T. 1991, ApJ, 372, 185

Lynds, B. T. 1962, ApJS, 7, 1

Meyers, K. A., Snow, T. P., Federman, S. R., \& Breger, M. 1983, ApJ, 288, 148

Obayashi, A., Fukui, Y., Kun, M., Sato, F., \& Yonekura, Y. 1998, AJ, 115, 247

Sancisi, R. 1974, in IAU Symposium 60, Galactic Radio Astronomy, ed. F. J. Kerr \& S. C. Simonson (Dordrecht: Reidel)

Seitter, W. C. 1975, Bonner Spectral Atlas II. (Ferdinand Dümmler Verlag)

Solomon, P. M., Rivolo, A. R., Barrett, J. W., \& Yahil, A. 1987, ApJ, 319, 730

Strong, A. W., \& Mattox, J. R. 1996, A\&A, 308, L21

Tafalla, M., Mardones, D., Myers, P. C., et al. 1998, ApJ, 504, 900

Taylor, D. K., Dickman, R. L., \& Scoville, N. Z. 1987, ApJ, 315,104

Tóth, L. V., Kiss, Cs., \& Moór, A. 1996, in The Role of Dust in the Formations of Stars ESO workshop, ed. H. U. Käufl (Springer), 125

Trumpler, R. J., \& Weaver, H. F. 1953, Statistical Astronomy (Univ. of California Press, Berkeley and Los Angeles)

Wainscoat, R. J., Cohen, M., Volk, K., et al. 1992, ApJS, 83, 111

Wheelock, S. L., et al. 1994, IRAS Sky survey Atlas Explanatory Supplement, JPL Publication 94-11, Pasadena:JPL

Wilson, T. L., \& Rood, R. 1994, ARA\&A, 32, 191

Wolf, M. 1923, AN, 219, 109

Yonekura, Y., Dobashi, K., Mizuno, A., et al. 1997, ApJS, 110,21 